\title{
Tomographic Diffractive Imaging. STEM Lithography for Nanorings.
}

\author{
J.C.H.Spence, U. Weierstall. \\ Dept of Physics, Arizona State University \\ Tempe, Az. 85282.
}

Nanoscience provides a unique opportunity for electron microscopy because, for individual particles, single scattering conditions can be expected and elastic relaxation due to thinning are absent. Fieldemission electron microdiffraction patterns from these particles provides the strongest signal from the smallest volume of matter known to Physics. The success of iterative algorithms with X-rays [1] and Light [2,3] for solving the phase problem then suggests that tomographic superesolution images should be obtainable by inversion of coherent electron diffraction patterns from individual particles, as first predicted in atomic-resolution simulations [2]. We have applied the Fienup hybrid inputoutput (HiO) algorithm [4] to Kohler-mode selected-area field-emission diffraction patterns from individual $\mathrm{MgO}$ cubes, from which images might be reconstructed at subatomic resolution in three dimensions (See $\mathrm{Wu}$ et al, these proceedings). TEM images are used to provide the support. As with X-ray work, the preparation of an isolated object of known support (boundary) is the greatest challenge in this work. In addition, the use of a probe to isolate a diffracting region creates a "complex object" for which $\mathrm{HiO}$ performs poorly (see [3] for a summary of convergence conditions). For a strong phase object, the unit modulus constraint in real space assists convergence.

For a slab with normal $\mathrm{z}$, we have also investigated the use of the compact support constraint along $\mathrm{z}$ as a general solution to the phase problem in the transmission geometry. Specifically, we apply the $\mathrm{HiO}$ algorithm to transmission electron diffraction from monolayer organic crystals which are periodic in directions normal to $\mathrm{z}$. We find that oversampling along the reciprocal-lattice rods along $z$ does not solve the phase problem in this geometry in general. Convergence in three-dimensions is obtained, however if a small number of known phases are used, as may be obtained at more limited resolution from electron microscope images of the same sample. This is understandable since a knowledge of phase is a convex constraint [5]. In this way the "missing cone" problem might be solved by applying the $\mathrm{HiO}$ algorithm to cryomicroscopy monolayer crystal diffraction data, and the number of orientations for which images are needed can be greatly reduced, with a consequent reduction in radiation damage. We study the resolution needed in the images for tomographic reconstruction at atomic resolution, and the reduction in radiation dose made possible by this method. Figure 1 shows a simulation. The kinematic electron diffraction pattern for a monolayer of linear silicon dioxide molecules has been calculated in three dimensions. This shows reciprocal lattice rods normal to the monolayer, and Bragg reflections laterally. We assume that only intensities can be recorded, and that these oversample the data along the rods with a spacing finer than the Shannon spacing for the "band-limit" imposed by the finite thickness $t$ of the film. (Since the diffracted intensity along $\mathrm{z}$ is the transform of the autocorrelation function of the shape function of the film, this spacing is $\Delta \theta / \lambda=1 /(2 \mathrm{t})$, not $1 / \mathrm{t})$. Three-dimensional HiO iterations are then 
implemented between real and reciprocal space, applying the known shape function, diffracted amplitudes, and sign of the scattering potential in each iteration. If phases are provided from images to $0.035 \mathrm{~nm}$ resolution on only two planes in reciprocal space through the origin (unrelated by symmetry), the algorithm converges in 180 iterations to the correct image shown in the figure. This solves the phase problem for this two-dimensionally periodic object, providing an image from the diffraction pattern alone, at $0.05 \mathrm{~nm}$ resolution.

In other work we have developed methods for lithographic formation of nanorings using the Vacuum Generators HB501S STEM in a direct-write, inorganic process (Jiang, Hembree et al, these proceedings, and [6]). Currently we are able to make germanium rings about $20 \mathrm{~nm}$ in diameter within a silicate glass film. The optical absorption has been calculated in collaboration with F. Garcia de Abajo. The rings may have useful superconductive (squid), quantum dot and/or photonic properties. Supported by awards from ARO and NSF.

\section{References.}

[1]. H. He et al, Acta Cryst. ( 2003). A: In press.

[2]. U. Weierstall Ultramic. 90 (2001) 171.

[3]. J. Spence et al Phil Trans. 360 (2002) 875.

[4]. R. Fienup, J. Opt Soc Am,. A4 (1987) 118.

[5]. S. Lindaas et al. in X-ray microscopy and spectroscopy. (1998) Springer. NY.

[6]. N. Jiang et al. Appl Phys Letts., 2003. submitted.




https://doi.org/10.1017/S1431927603440671 Published online by Cambridge University Press 